\title{
DOE/PC/ $91334-785$
}

DOE/PC/91334--T85

DE93 000534

\section{TECHNICAL REPORT}

March 1, 1992 through May 30, 1992

Project Title: Protocols for the Selective cleavage of Carbon-Sulfur

Bonds in Coal

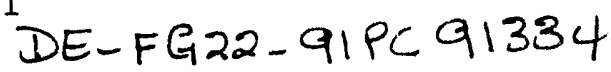

Principal Investigator: Mark Bausch, Southern Illinois University

Project Monitor: Dr. Ken K. Ho, CRSC

\section{ABSTRACT}

Chemical reactions that result in carbon-sulfur bond cleavage are an essential aspect of any protocol designed to remove organjc sulfur from coal. Unfortunately, several classes of reactions that lead to carbonsulfur bond cleavage are not well understood. Planned in "Protocols for the Selective Cleavage of Carbon-Sulfur Bonds in Coal" are reactions in which organic sulfur-containing coal model compounds are subjected to different conditions of temperature, solvent mixtures and radiation. Summarized in this quarterly report are results of our investigations of the following topics: (a) the reactions of coal model compounds, namely, benzyl phenyl sulfide (BPS), diphenyl sulfide (hereafter referred to as phenylsulfide, PS) and dibenzothiophene (DBT) with various reagents (Lewis acid catalysts, radical initiators, electron acceptors) using different solvents and temperature in an attempt to maximize the degree of carbon-sulfur (C-S) bond cleavage; and (b) the results of photooxidation of coal model compounds under controlled conditions. Quantitative product analyses are presented in this report.

\section{DISCLAIMER}

This report was prepared as an account of work sponsored by an agency of the United States Government. Neither the United States Government nor any agency thereof, nor any of their employees, makes any warranty, express or implied, or assumes any legal liability or responsibility for the accuracy, completeness, or usefulness of any information, apparatus, product, or process disclosed, or represents that its use would not infringe privately owned rights. Reference herein to any specific commercial product, process, or service by trade name, trademark, manufacturer, or otherwise does not necessarily constitute or imply its endorsement, recommendation, or favoring by the United States Government or any agency thereof. The views and opinions of authors expressed herein do not necessarily state or reflect those of the United States Government or any agency thereof.

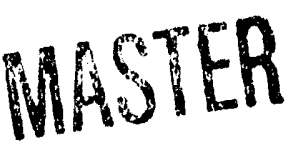
DISTRIBUTION OF THIS DOCUMENT IS UNLIMTVE.

This project is funded by the U. S. Department of Energy (PETC) and by the Illinois Department of Energy and Natural Resources as part of their costoh: rat nrogramo 


\section{EXECUTIVE SUMMARY}

The following sets of experiments were slated for completion during the two-year funding period for "Protocols for the selective cleavage of Carbon-Sulfur Bonds in Coal":

1. Allow various coal model compounds (species that contain aliphatic and aromatic $\mathrm{C}-\mathrm{S}$ bonds) to be subjected to various conditions. The variables in these studies include different substrates, solvents, bases, added reagents (catalysts, oxidizing agents, electron acceptors), temperature and pressure. Photochemical reactions of these coal model compounds will also be studied by varying the wavelength of light, using various solvents with or without sensitizers, oxidizing the compounds and subsequently hydrolyzing the products of the reaction.

2. Subject coal and coal model compounds to ultrasonic irradiation for different periods of time. The effects of adding catalysts or oxidizing agents will also be determined. Different substrates, solvents and oxidizing agents, along with ultrasonic irradiation, altogether will form a matrix of results that will allow determination of optimum conditions that will accomplish the desired $c-s$ bond cleavage on coal samples.

-3. Heat the coal model compounds containing both aliphatic and aromatic sulfur, oxygen, carbon, and nitrogen at various temperatures, using a Differential Scanning Calorimeter(DSC). Observe the transitions in the sample brought about by $\mathrm{C}-\mathrm{S}, \mathrm{C}-\mathrm{O}, \mathrm{C}-\mathrm{C}$ and $\mathrm{C}-\mathrm{N}$ bond cleavages.

-4. Investigate differences in the bond strengths in the radical cations derived from coal model compounds 10-phenylthio-9-methylanthracene and 9-phenylthiomethylanthracene. We will attempt to ascertain the differences in reactivities of different types (i.e. aromatic or aliphatic carbon) of carbon-sulfur bonds, under various reaction conditions, and correlate these differences with their respective bond strengths.

As described in this quarterly report, our efforts have been concentrated in the areas of the first bullet. During the past 3 months (March 1, 1992 - May 30, 1992), experiments were carried out in which coal 
model compounds BPS, PS and DBT (with or without radical initiators, base, metal/metal ion catalysts or electron acceptors) were subjected to reflux and bomb concitions $\left(320-340^{\circ} \mathrm{C}\right)$. The results indicate that:

- The bomb reactions yield more degradation products than the reflux reactions.

- For both reflux and bomb conditions, it was found that BPS most often yielded products arising from $\mathrm{C}-\mathrm{S}$ bond cleavage or oxidation; while PS and DBT were less likely to undergo C-S bond cleavage. Most of the reactions that involve BPS yield more products, than those that involve PS or DBT, which both contain only aromatic C-S bonds. These results lend support to the BDE values for aliphatic and aromatic C-S bonds, which were reported in previous quarterly reports.

- Among the solvents, 2-pyrrolidinone gave the most number of reactions that gave positive results ( $\mathrm{C}-\mathrm{s}$ bond cleavage) under reflux conditions, while the rest of the solvents gave about the same number of reactions that yield positive results. Nitrobenzene did not cause any cleavage nor oxidation reactions. On the other hand, quinoline makes up $21 \%$ of the reactions that gave positive reactions under bomb condilions. The decreasing efficiency for solvents in this study (under bomb conditions) to affect $\mathrm{C}-\mathrm{S}$ bond cleavage is as follows: quinoline > THF=morpholine > decalin > 2-pyrrolidinone > nitrobenzene > 1-methyl-2-pyrrolidinone. - Lewis-acid catalyzed reactions account for 408 of the reactions that produce positive results when conducted under reflux conditions; solvent-mediated (25\%); electron-acceptor (fluorenone or benzoquinone)assisted (15\%); base-mediated (10\%) and AIBN (azobisisobutyronitrile) initiated $(108)$. Under bomb conditions, product conversions were in the following order: electron acceptor-assisted reactions > Lewis-acid catalyzed > AIBN-initiated > base-mediated > and solvent-mediated.

Photooxidation of BPS for three hours resulted in the formation of benzyl phenyl sulfone as the major product and benzaldehyde as a minor product. Under identical conditions, DBT formed $37 \%$ dibenzothiophene sulfoxide after 24 hours of irradiation. $50 \%$ of benzothiophene remained unreacted after 21 hours of irradiation. Complex products that are difficult to characterize are obtained upon photooxidation of thiophene. Isolation and identification of the reaction products is ongoing. 
Our long range objective in the research funded by CRSC has been to carry out experiments that will aid in solving the problem of coal desulfurization--from the perspective of inventing, understanding, and perfecting new carbon-sulfur bond breaking chemical reactions. Experiments carried out during the past three months included studies of reactions in which radical initiators, metal ions, base mixtures and electron acceptors were allowed to react with sulfur-containing coal model compounds under refluxing and high temperature (Parr bomb) conditions. Other experiments carried out during this reporting period included studies of photochemical and photo-oxidation reactions of sulfur-containing coal model compounds in the presence/absence of sensitizers. It is our objective to develop a set of low-temperature reaction conditions that will enable substantial $\mathrm{C}-\mathrm{S}$ bond cleavage to be affected in Illinois coal. The described experiments are the first steps in achieving some understanding at the molecular level of a newly-invented process.

Examination of the four tasks listed below reveals details of the experiments planned to aid in the efforts to reach the stated objective:

TAsK 1:Carry out experiments that aim to selectively cleave C-S bonds in coal model compounds benzyl phenyl sulfide, phenyl sulfide and dibenzothiophene, as well as physically cleaned Illinois coal. Main thrusts of these investigations include radical-and Lewis acidinitiated, as well as caustic- and photooxidatively-assisted C-S bond cleavages.

TASK 2: Evaluate the effect of ultrasonic radiation on the effectiveness of several C-S bond cleavage protocols.

TAsK 3:Utilize differential scanning calorimetry (DSC) techniques in examinations of various methods of cleaving $\mathrm{C}-\mathrm{S}$ bonds in coal model compounds, as well as gaining understanding of the degree of depolymerization in Illinois coals (coals that contain varying amounts 
of organic and inorganic sulfur) when these coals are subjected to depolymerization protocols. Also planned are DSC studies of several Illinois coals subjected to a variety of pretreatment conditions.

TASK 4:Carry out experiments that enable determinations of the bond energies for aliphatic and aromatic $\mathrm{C}-\mathrm{S}$ bonds in the radical ions derived from several coal model compounds. Communicate these results to the coal-desulfurization community through publications in scientific journals.

Contained in this quarterly report are summaries on our efforts concerning TASK 1 along with selectcd other results.

INTRODUCTION AND BACKGROUND

The organic sulfur found in coal consists primarily of sulfur atoms covalently bonded to aliphatic or aromatic carbon atoms contained in the backbone of the coal macromolecule (there may be a small percentage of $S-S$ and $S-H$ bonds in coal as well). The sulfur present in Illinois coal is problematic because the combustion of high-sulfur coal results in the formation of species implicated in environmental problems related to acid rain. The relevance and significance of the experiments planned for the present funding period is perhaps best described by acknowledgment of the following two statements:

(a) In order to remove the organic sulfur from coal, carbon-sulfur bonds must be broken.

(b) Chemists' knowledge and awareness of reactions that lead to the selective cleavage of carbon-sulfur bonds, and in particular, sulfur atoms bonded to $\mathrm{sp}^{2}$ (i.e. aryl) carbon atoms, is limited.

The experiments outlined in the four TASKS listed earlier in this quarterly report are aimed, in the short term, at developing an increased understanding of methods that enable cleavage of $\mathrm{C}-\mathrm{S}$ bonds. The longer term goal of these experiments is the invention of new chemical reactions, or perfection of existing chemical reactions. The attainment of reaction conditions that allow the selective cleavage of 
carbon-sulfur bonds in coal and coal model compounds is therefore the main goal of "PROTOCOLS FOR THE SELECTIVE CLEAVAGE OF CARBON-SULFUR BONDS IN COAL". An important outcome of these studies is the resulting increase in chemists' knowledge of carbon-sulfur bond cleavage reactions. Successful completion (successful in terms of affecting carbon-sulfur bond cleavages) of the studies deccribed in this proposal thus increases the likelihood of the deployment of a simple and costeffective protocol designed to remove organic sulfur from Illinois coal. Much of the coal mined in Illinois suffers from its high sulfur content. Methods have been developed that are quite effective at removing the inorganic sulfur from coal. Unfortunately, the problem of organic sulfur present in Illinois coal remains to be solved. A major reason for the lack of progress in the area of organic sulfur removal is the lack of knowledge concerning carbon-sulfur bond cleavage reactions. completion of the experiments outlined in TASKS 1-4 will at least partly remedy this situation. It is our desire to convert what we learn from these studies of carbon-sulfur bond breaking reactions into a simple protocol that will offer a reasonable and economically viable solution to the problems of using Illinois coal.

The experiments summarized in this quarterly report are best described as fundamental studies of carbon-sulfur bond cleavage reactions, using both coal and model compounds. At present, none of these experiments describe a process that is cost-effective in terms of removing organic sulfur from coal. It is our desire to develop a practical carbon-sulfur bond-breaking technology from the research described in this proposal, and transition this new technology to the Illinois coal community.

EXPERIMENTAL PROCEDURES

TASKS 1-4 describe experiments that have synthetic, colorimetric, and electrochemical components. The synthetic component of our work is typical of the procedures that practicing organic chemists follow. A uv-visible spectrophotometer is utilized in the collection of appropriate acid-base and kinetic data. A differential scanning 


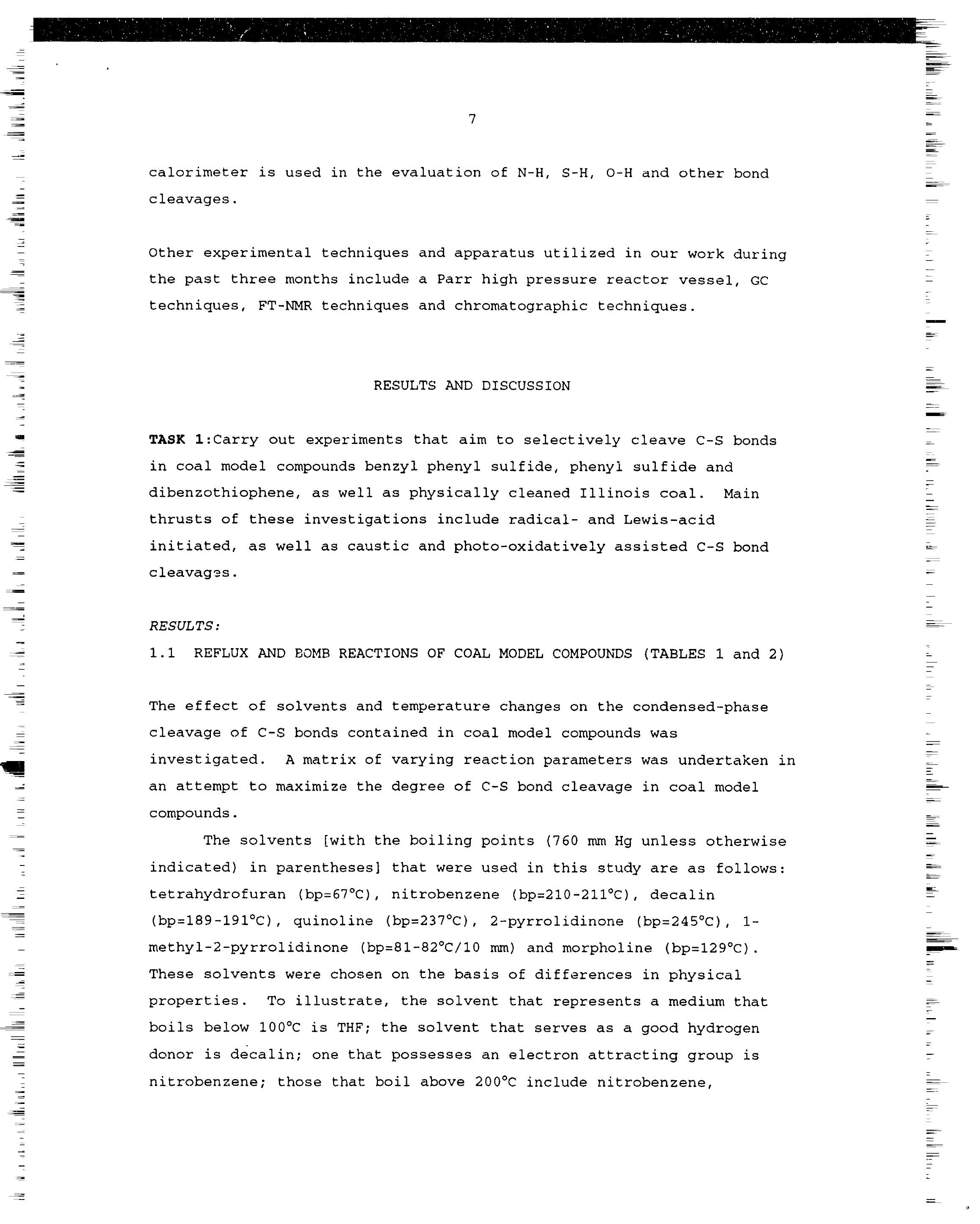


quinoline, 1-methyl-2-pyrrolidinone and 2-pyrrolidinone; while the medium that could be heated up above $100^{\circ} \mathrm{C}$ but below $200^{\circ} \mathrm{C}$ is morpholine. Reflux conditions involve heating the reaction mixture at the boiling point of the solvent for four days and then analyzing the mixture by TLC, NMR and GC. A Parr reactor was used for reactions that were heated up to a temperature of $310-340^{\circ} \mathrm{C}$ for three hours.

The quantitative results that were presented in the previous quarterly report were preliminary in the sense that the data were based on marual mieasurement of the height and width of each peak using a ruler. The calculated areas are therefore rough approximations of the relative amounts of products formed in each reaction. The concentrations of each product are determined using calibration curves of standard compounds that are predicted to have formed during the reactions. Products are analyzed with TLC, GC and NMR. Our newlyacquired integrator allows us to quantitate the products more accurately. The results presented in Table 1 are based on the relative areas of the peaks in the chromatogram, excluding the solvents. Most of these reaction mixtures contain a charred polymeric material which is insoluble in any organic solvent and is difficult to identify and characterize. The polymeric material, by mass, is typically one-quarter to one-third of total mass of the starting sulfide. Mass balances for the reactions are typically greater than $75 \%$. The column on "Product Analysis" is a listing of the identified products derived from each reaction, with the corresponding relative percentage areas. Unidentified products account for the balance of the percentage areas.

Based on the data in Table 1, the following inferences can be made: - Among the seven solvents that were evaluated, 2-pyrrolidinone was most effective in terms of its ability to affect $\mathrm{C}-\mathrm{S}$ bond cleavage. Out of the 20 reactions that yielded isolable products, 9 reactions occurred in the presence of 2-pyrrolidinone, namely, reactions $2,3,4,5,10,11,14,15$ and 16. An apparent indication of $C-S$ bond cleavage is detected for BPS as substrate, as shown in the formation of dibenzyl, diphenyl disulfide, toluene and benzaldehyde. The formation of benzyl phenyl sulfone is an indication that oxidation is also occurring in some of these reactions. 
For PS and DBT substrates, other products are formed as detected by gas chromatography, TLC and NMR. As of this writing, these products are still unidentified.

One of the factors that could explain the lability of the C-S bonds in a reaction involving 2-pyrrolidinone (relative to the other solvents) is the refluxing temperature. 2-Pyrrolidinone is among the highest boiling solvents that were used in these experiments.

- Comparison of AIBN-initiated reactions and non-AIBN-initiated reactions, such as 1 vs. 18 and 2 vs. 16 shows that the presence of a radical-initiator may or may not contribute significantly to the reactivity of the substrate. For instance, in reactions ( 1 and 18 ) utilizing 1-methyl-2-pyrrolidinone as the solvent, the presence of AIBN substantially lowers the amount of unreacted BPS. However, in reactions (2 and 16) that involve 2-pyrrolidinone as solvent, no significant change in unreacted starting material is observed when AIBN was added to the mixture.

- The use of $\mathrm{ZnCl}_{2}$ catalyst in refluxing solvents resulted in the formation of $\mathrm{C}-\mathrm{S}$ bond cleavage products such as dibenzyl and phenyl disulfide. About 40 and $80 \%$ benzaldehyde was observed when BPS was refluxed with 2-pyrrolidinone and morpholine.

- The reaction conducted in the presence of an electron acceptor such as 1,4 -benzoquinone, as in reaction 13, has a reduced amount of unreacted BPS compared to the reaction without 1,4-benzoquinone, as in reaction 17. The addition of benzoquinone resulted to the formation of dibenzyl, benzaldehyde and benzyl phenyl sulfone. On the other hand, the products observed in reaction 17 , are the following: dibenzyl (2.2\%), benzaldehyde (6.48), diphenyl disulfide (14.2\%) and toluene (0.6\%). The other benzoquinone-assisted reactions did not show any signs of bond cleavage.

The following are the results and discussion for the bomb reactions (Table II) :

Reactions 1-10: AIBN-initiated reactions. The amount of unreacted BPS in these reactions is small (typically $<28$ ). Products that are formed 
as a consequence of $\mathrm{C}-\mathrm{S}$ bond cleavage reactions are toluene, dibenzyl, benzaldehyde and benzoic acid. Phenyl sulfide is formed in varying amounts (2-63\%) upon heating BPS with AIBN in different solvents. The reaction of $P S$ and $D B T$ with $A I B N$ results in the formation of very low amounts of DBT sulfone and sulfoxides.

Reactions 11-19: Addition of a 1:1 mixture of $\mathrm{NaOH}$ and $\mathrm{KOH}$ to $\mathrm{BPS}$ appears to induce oxidation processes. Formation of benzyl phenyl sulfone ranges from 5-99\%. Lower conversions are observed for PS and DBT substrates. The presence of dibenzothiophene sulfoxide accounts for about 3-15\% of identified products for the DBT + base reactions.

Reactions 20-28: Solvent-mediated reactions. Pnenyl sulfide is formed in largest amounts for the substrate BPS in solvents such as decalin, 1-methyl-2-pyrrolidinone, morpholine and quinoline. A small amount of phenyl sulfide is obtained using 2-pyrrolidinone as the solvent. Another product that is common to the solvent-mediated BPS experiments is benzyl phenyl sulfone. The amount of unreacted BPS is low. For PS and DBT substrates, only $1-2 \%$ of the products are identifiable by GC and NMR. The product that is common to all three reactions involving aromatic sulfides, namely reactions $25-27$, is biphenyl (< 18 ). In order for tinis product to be formed, the sulfur atom has to be removed as hydrogen sulfide. Among the various conditions employed in these experiments, the solvent-mediated reactions had the worst smell of rotten egg after termination of the bomb run.

Reactions 29-35: Phenyl sulfide was found to occur as a major product for reactions 29,30 and 33 . These trials involve $\mathrm{ZnCl}_{2}$ as the catalyst and quinoline, morpholine and nitrobenzene as solvents. Other solvents such as THF and decalin (reactions 31 and 32 ) also yield phenyl sulfide but in low yields. The major product for these reactions is dibenzyl. As in the other experiments that involve substrates that contain aromatic sulfides such as DBT and PS, the per cent conversion is below 28. It was only in morpholine solvent that apparent $\mathrm{C}-\mathrm{S}$ bond cleavage occurred upon reacting $\mathrm{ZnCl}_{2}$ and $\mathrm{DBT}$ or PS. 
Reactions 36-40: For reaction 37, a high yield of phenyl sulfide is formed upon reacting BPS with $\mathrm{SnCl}_{2}$ in decalin. Other products for this reaction included benzyl phenyl sulfoxide, and in smaller amounts, benzaldehyde, dibenzyl, and benzyl phenyl sulfone. For the $\mathrm{SnCl}_{2}-$ mediated reaction of BPS in THF, the major product is benzyl phenyl sulfone, while in quinoline and 2-pyrrolidinone the major product is phenyl sulfide. Among all the reactions conducted for aromatic sulfide-containing substrates, only phenyl sulfide in quinoline formed trace amounts of $\mathrm{C}-\mathrm{S}$ bond cleavage products such as thiophenol and biphenyl.

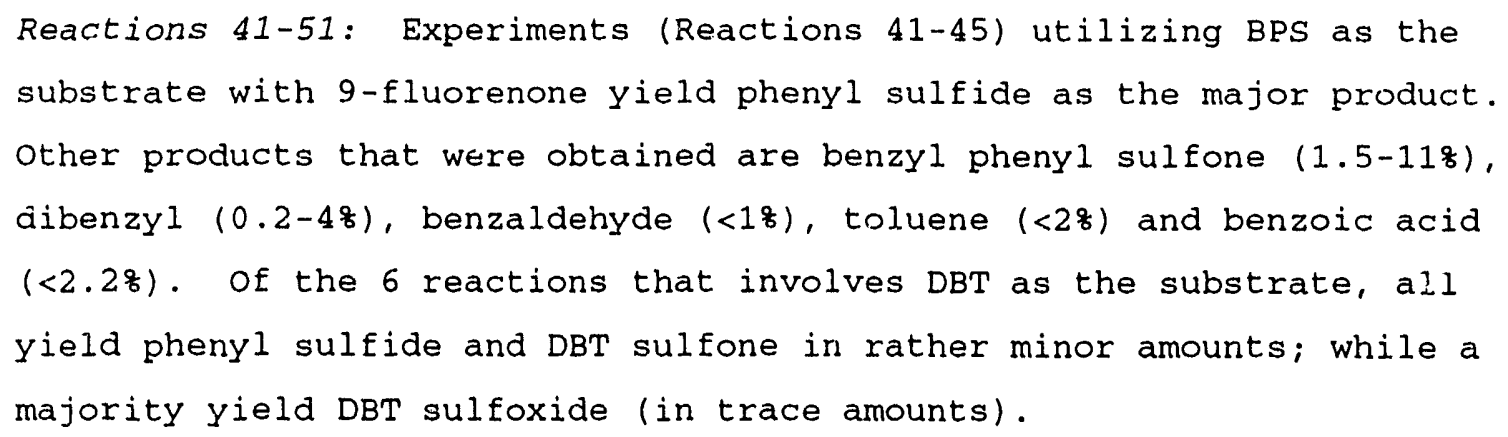

summary. Based on the data on Tables 1 and 2 ,

- The conditions in the bomb reactions are more drastic and consequently, give more degradation products than the reflux reactions. - Among the solvents, 2-pyrrolidinone was shown to be the "best" solvent under reflux conditions, while the rest of the solvents gave about the same number of reactions that yield positive results. Nitrobenzene did not cause any bond cleavage nor oxidation reactions. On the other hand, quinoline makes up $21 \%$ of the reactions that gave positive reactions 
under bomb conditions. The decreasing order by which solvents play a role in bond cleavage under bomb conditions is as follows: quinoline > THF=morpholine > decalin > 2-pyrrolidinone > nitrobenzene > 1-methyl-2pyrrolidinone.

- Lewis-acid catalyzed reactions account for $40 \%$ of the reactions that produce positive results when conducted under reflux conditions; solvent-mediated (25\%); electron-acceptor (fluorenone or benzoquinone)assisted (15\%); base-mediated (10\%) and AIBN-initiated (10\%). Under bomb conditions, the following gives the decreasing order of product conversion: electron acceptor-assisted reactions (28.5\%); Lewis-acid catalyzed (21\%); AIBN-initiated (18\%); base-mediated (168) and solventmediated $(16 \%)$.

\subsection{SUPEROXIDE OXYGENATION OF ORGANIC SULFIDES}

The same light source (Hanovia lamp - 654A36, filtered through Pyrex) and standard photochemical reactor were used as described in previous experiments. Oxygen was bubbled through $150 \mathrm{ml}$ of the $0.01 \mathrm{M}$ solution of sulfide in acetonitrile. Anthracene concentration was $0.0001 \mathrm{M}$. Photooxidations were monitored by GC and TLC. Products were isolated by column chromatography (silica gel, with chloroform-hexane mixtures as eluents) and characterized by GC, TLC and ${ }^{1} \mathrm{H}$ and ${ }^{13} \mathrm{C}$ NMR (DMSO-d6 solution). Four compounds have been studied: benzyl phenyl sulfide, dibenzothiophene, benzothiophene and thiophene. The following are the results of our studies of the photoxidative reactivities of coal model compounds :

Benzyl phenyl sulfide. The reaction is completed after about three hours. GC indicates only $0.1 \%$ of the initial amount of the substrate and at least 10 other substances present in the reaction mixture. Interpretation of NMR spectra of the product is difficult because of the large number of signals. However, it was possible to identify three compounds: benzyl phenyl sulfoxide, benzyl phenyl sulfone, and benzaldehyde. These findings were supported by GC and TLC. It is important to note that NMR spectra of the product mixture were different 
from those observed for 9,10-dicyanoanthracene (DCA) sensitized photooxidation (e. g. strong signal at $6.1 \mathrm{ppm}$ on $1_{H}$ NMR spectrum is observed only for anthracene sensititized reaction).

Column chromatography allowed isolation of $70 \mathrm{mg}$ of the $1: 2$ mixture sulfoxide-sulfone (estimated by integration of the ${ }^{1}$ H NMR signals). No other compounds were isolated in a form allowing quantitative estimations.

The above reaction is slower than the DCA sensitized process and leads to different products (benzyl phenyl sulfone seems to be the major oxidation product). Benzaldehyde is present but estimation of the extent of $C-S$ bond clravage is impossible at this time since separation of the complex mixture of products is very difficult.

Dibenzothlophene. As we reported previously, after 24-hours reaction there is still a small amount of the substrate present in the reaction mixture (the corresponding peak is present on gas chromatogram). The major product was isolated by column chromatography and identified as dibenzothiophene sulfoxide. The yield of the sulfoxide was about $37 \%$. This compound was identified by comparison of its melting point and NMR spectra with those recorded for an original sample of sulfoxide, prepared by $\mathrm{H}_{2} \mathrm{O}_{2}$ oxidation of dibenzothiophene. The other product, dibenzothiophene sulfone, was isolated as well (yield:11\%). This is an interesting result in light of our observation that the DCA sensitized reaction produced sulfone as a major isolated product. However, all of the sulfoxide signals are present on the ${ }^{13} \mathrm{C}$ NMR spectrum of the crude product of the DCA sensitized oxidation. Since sulfoxide peak on GC cannot be clearly resolved from that of sulfone it is possible that this substance was present in the mixture in a significant amount but was not eluted during column chromatography (sulfoxide is eluted very slowly even by pure chloroform). More experiments are necessary to evaluate this possibility.

Benzothlophene After about 5 hours of irradiation concentration of the substrate in the reaction mixture remained unchanged (GC). After 21 hours there was still about $50 z$ of benzothiophene present, with no other 
peaks observed on the gas chromatogram. There is no separation by TLC, except for the starting material. Most of the signals observed on $1_{\mathrm{H}}$ and ${ }^{13} \mathrm{C}$ NMR spectra can be found on those of the product of the DCA sensitized reaction.

Thlophene After 7 hours of irradiation thiophene cannot be smelled in the reaction mixture and is not detected by TLC. After evaporation of solvent only about $20 \mathrm{mg}$ of brown oil remained. This is only $15 \%$ of the mass of the substrate. It is possible that thiophene and/or some volatile products evaporated during the reaction leaving only highmolecular-weight products that do not show any peaks on GC and cannot be separated by TLC. The ${ }^{1} \mathrm{H}$ NMR spectrum is complicated and difficult to analyze. However, the ${ }^{13} \mathrm{C}$ NMR spectrum is relatively simple. There are at least 15 spectral lines on the spectrum but two of them (at 134.5 and $126.7 \mathrm{ppm}$ ) are more than 10 times stronger than others, another relatively strong signal is observed at $171.4 \mathrm{ppm}$. None of these signals were observed for the product of DCA sensitized reaction.

TASK 2:Evaluate the effect of ultrasonic radiation on the effectiveness of severa' C-S bond cleavage protocols.

RESULTS: No experiments were undertaken for this task for this period.

TAsK 3:Utilize differential scanning calorimetry (DSC) techniques in examinations of various methods of cleaving $C-S$ bonds in coal model compounds, as well as gaining understanding of the degree of depolymerization in Illinois coals (coals that contain varying amounts of organic and inorganic sulfur) when these coals are subjected to depolymerization protocols. Also planned are DSC studies of several Illinois coals subjected to a variety of pretreatment conditions.

RESULTS:No experiments were undertaken for this task for this period.

TASK 4: Carry out experiments that enable determinations of the bond energies for aliphatic and aromatic $c-s$ bonds in the radical ions 
derived from several coal model compounds. Communicate these results to the coal-desulfurization community through publications in scientific journals.

RESULTS: We cortinue to receive dozens of requests for reprints for our publications in Energy \& Fuels, the Journal of the American Chemical Society, Polycyclic Aromatic Compounds, and the Journal of Organic Chemistry. Many of these articles focus on evaluations of bond strengths in molecules likely to be found in the backbone of coal macromolecules.

Determinations of bond strengths for $\mathrm{C}-\mathrm{S}$ bonds present in radical cations derived from coal model compounds are in progress and will be reported on in a future summary. 
TABLE 1. Product analysis for the reflux reactions. Yields are relative and based on gc analyses.

1. BPS + AIBN + 1-Me-2-pyrrolidinone BPS ( 53.38 )

Bipheny 1 (37.98)

Phenyl sulfoxide (7.78)

AIBN (1.08)

2. BPS + AIBN + 2-pyrrolilinone BPS ( 52.08$)$

Dibenzyl (25.68)

Benzaldehyde (19.48)

Benzyl phenyl sulfone (3.2\%)

3. PS + base + 2-pyrrolidinone

PS (81.4\%)

Dibenzyl (18.68)

4. $\mathrm{DBT}+$ base + 2-pyrrolidinone DBT (72.18)

5. BPS $+\mathrm{SnCl}_{2}+2$-pyrrolidinone BPS (79.8\%)

Dibenzyl (16.28)

Benzyl phenyl sulfone $(0.68)$

6. BPS $+\mathrm{SnCl}_{2}+$ quinoline

BPS $(24.4 \%)$

7. $\mathrm{DBT}+\mathrm{SnCl}_{2}+$ quinoline

DBT $(68.48)$

8. $\mathrm{PS}+\mathrm{SnCl}_{2}+$ quinoline PS (298)

9. $\mathrm{BPS}+\mathrm{ZnCl}_{2}+\mathrm{THF}$ BPS (81.0\%)

Dibenzyl (9.5\%)

10.BPS $+\mathrm{ZnCl}_{2}+2$-pyrrolidinone

BPS (57.3\%)

Benzaldehyde (39.8z)

Phenyl disulfide $(2.88)$

11.DBT $+\mathrm{ZnCl}_{2}+2$-pyrrolidinone

DBT $(96.18)$

Benzaldehyde ( $3.8 \%)$
12.BPS $+\mathrm{ZnCl}_{2}+$ morpholine Benzaldehyde (80z)

BPS (20\%)

13.BPS + benzoquinone + THF

BPS $(46.3 \%)$

Dibenzyl (21.38)

Benzaldehyde (13.08)

Benzyl phenyl sulfone (20.0\%)

14.PS + 2-pyrrolidinone

PS (82.8\%)

15.DBT + 2-pyrrolidinone

DBT (2.8\%)

Benzyl phenyl sulfoxide (35.18)

16.BPS + 2-pyrrolidinone

BPS (438)

Toluene (43\%)

Dibenzyl (13.3\%)

Benzyl phenyl sulfone (1.4\%)

17. BPS + THF

BPS (72.5\%)

Diphenyl disulfide (14.2\%)

Benzaldehyde ( $6.4 \%)$

Toluene $(0.6 \%)$

Dibenzyl (2.28)

18.BPS + 1-Me-2-pyrrolidinone

BPS (88.8\%)

Dibenzyl (6.5\%)

Benzaldehyde (1.18)

Diphenyl disulfide (3.68)

19. DBT + fluorenone + morpholine DBT $(94.38)$

20.PS + fluorenone + morpholine

PS (71.0ㅇ)

Fluorenone $(0.98)$ 
TABLE 2 Product Analysis for the Bomb Reactions. Yields are relative and based on $\mathrm{gc}$ analyses.

1. $B P S+A I B N+T H F$

Toluene (3.4\%)

Benzaldehyde $(0.68)$

Dibenzyl (3.28)

PS (21.8\%)

BPS (0.4\%)

Benzoic acid (2.08)

Benzyl phenyl sulfone (1.2\%)

2. BPS + AIBN + decalin

PS $(2.68)$

3. BPS + AIBN + nitrobenzene

Dibenzyl $(0.38)$

PS (32.08)

Benzyl phenyl sulfone (1.0\%)

4. BPS + AIBN + quinoline

Toluene $(0.01 \%)$

Benzaldehyde (2.18)

Dibenzy 1 ( 3.48$)$

PS (63.18)

Benzyl phenyl sulfone (2.08)

AIBN $(0.38)$

5. BPS + AIBN + 2-pyrrolidinone

Dibenzyl (1.2\%)

PS $(34.6 \%)$

BPS (2.08)

Benzyl phenyl sulfone (2.6\%)

Benzaldehyde (2.0\%)

AIBN (0.48)

6. BPS + AIBN + 1-Me-2-pyrrolidinone

Dibenzyl (1.98)

PS (55.3\%)

Benzyl phenyl sulfone (1.28)

Benzaldehyde (1:18)

Benzoic acid (0.98)
$\operatorname{AIBN}(0.2 \%)$

7. BPS + AIBN + morpholine

Benzaldehyde (1.18)

Dibenzyl (1.68)

PS (45.8\%)

Benzyl phenyl sulfone (2.48)

Benzyl phenyl sulfoxide $(0.4 \%)$

AIBN $(0.3 \%)$

8. $\mathrm{PS}+\mathrm{AIBN}+\mathrm{THF}$

Phenyl sulfoxide $(0.6 \%)$

Benzene $(0.004 \%)$

9. $\mathrm{DBT}+\mathrm{AIBN}+$ nitrobenzene

DBT sulfone $(0.1 \%)$

PS $(0.18)$

DBT sulfoxide $(0.8 \%)$

10.DBT + AIBN + quinoline

Thiophenol $(0.18)$

UBT sulfone $(0.38)$

PS $(0.7 \%)$

AIBN $(0.4 \%)$

11.BPS + base + THF

Dibenzyl $(0.1 \%)$

PS $(0.18)$

BPS $(0.02 \%)$

Benzyl phenyl sulfone (99.1영)

12.BPS + base + nitrobenzene

Toluene $(0.28)$

Benzaldehyde (0.98)

Dibenzyl (1.4\%)

PS (11.8\%)

BPS $(0.48)$

Benzyl phenyl sulfone $(69.0 \%)$

Benzoic acid (0.4\%)

13.BPS + base + quinoline

Dibenzy 1 (0.5\%)

PS $(0.18)$

BPS $(0.5)$

Benzyl phenyl sulfone (98.9\%)

14.BPS + base + morpholine 


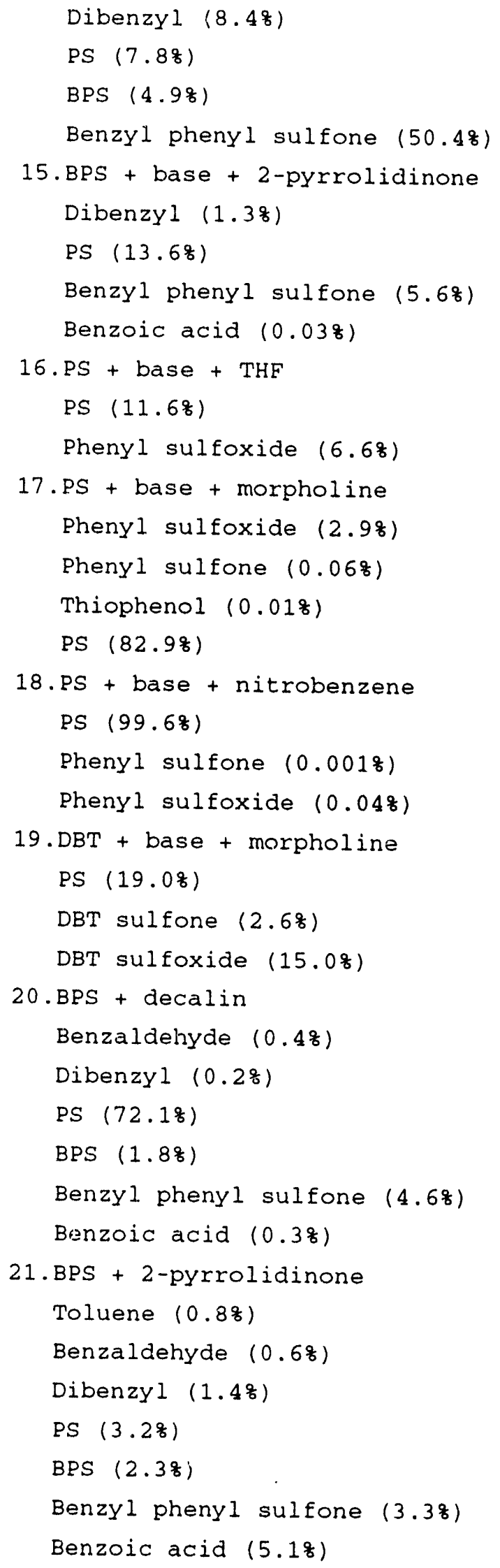

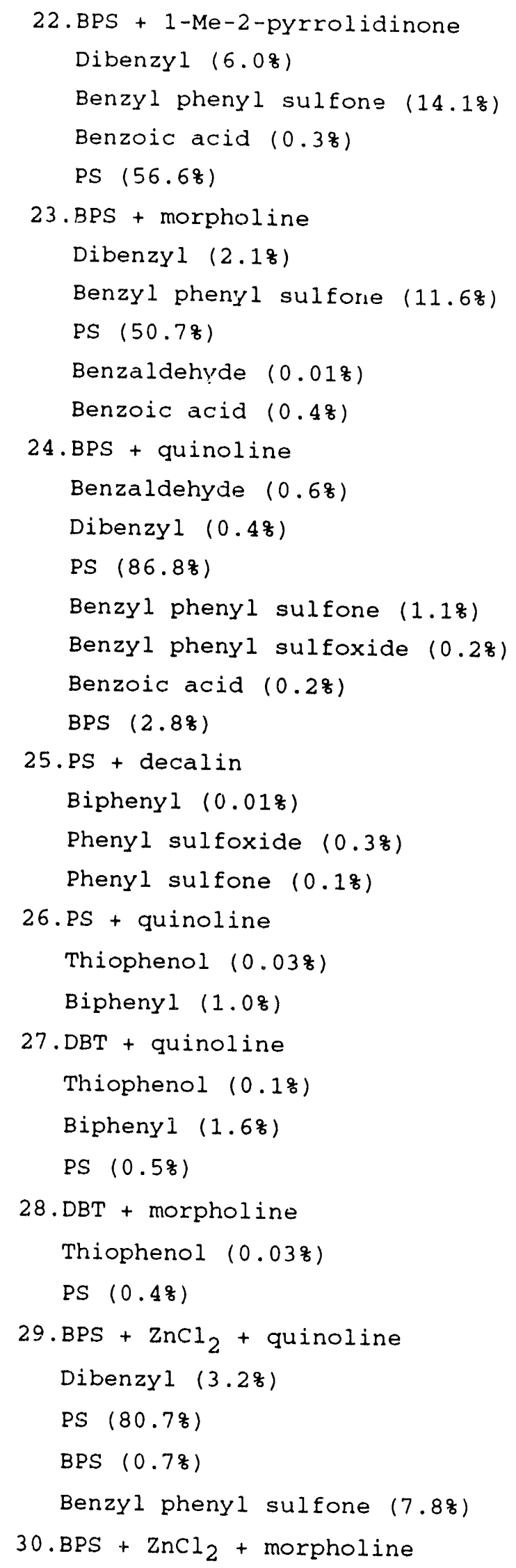


Benzaldehyde $(0.38)$

Dibenzyl (2.08)

PS $(55.2 \%)$

BPS $(0.7 \%)$

Benzyl phenyl sulfone ( $3.7 \%$ )

31. BPS $+\mathrm{ZnCl}_{2}+\mathrm{THF}$

Dibenzyl (46.78)

PS (7.5\%)

Benzoic acid $(0.88)$

$32 . \mathrm{BPS}+\mathrm{ZnCl}_{2}+$ decalin

Benzaldehyde (4.3\%)

Dibenzyl (47.38)

PS $(2.9 \%)$

BPS (3.5\%)

Benzyl phenyl sulfone (9.2z)

33. BPS $+\mathrm{ZnCl}_{2}+$ nitrobenzene

Dibenzyl (1.7\%)

Benzoic acid $(0.48)$

PS $(30.08)$

34.PS $+\mathrm{ZnCl}_{2}+$ morpholine

Phenyl sulfoxide $(0.1 \%)$

Thiophenol (0.003\%)

35.DBT $+\mathrm{ZnCl}_{2}+$ morpholine

DBT sulfoxide $(0.2 \%)$

DBT sulfone $(0.1 \%)$

Biphenyl $(0.1 \%)$

Thiophenol (0.003\%)

PS (1.4\%)

36. BPS $+\mathrm{SnCl}_{2}+\mathrm{THF}$

Benzaldehyde $(0.2 \%)$

Dibenzyl (6.88)

PS $(8.7 \%)$

BPS (1.1\%)

Benzyl phenyl sulfone (18.2\%)

Benzoic acid ( $1.7 \%$ )

$37 . \mathrm{BPS}+\mathrm{SnCl}_{2}+$ decalin

Benzaldehyde $(0.04 \%)$

Dibenzyl $(0.6 \%)$

PS $(76.38)$
$\operatorname{BPS}(0.4$ 웅)

Benzyl phenyl sulfone $(0.8 \%)$

Benzyl phenyl sulfoxide (16.58)

38. BPS $+\mathrm{SnCl}_{2}+$ quinoline

Toluene $(0.1 \%)$

Benzaldehyde $(0.38)$

Dibenzyl (5.0\%)

PS $(30.5 \%)$

BPS (11.2\%)

Benzoic acid (0.1\%)

30.BPS $+\mathrm{SnCl}_{2}+2$-pyrrolidinone

Benzoic acid (0.3\%)

PS (4.68)

40. PS $+\mathrm{SnCl}_{2}+$ quinoline

Thiophenol $(0.0048)$

Bipheny $1(0.68)$

PS $(90.2$ \%)

41.BPS + 9-fluorenone + THF

Benzaldehyde $(0.2 \%)$

Dibenzyl $(0.2 \%)$

PS (698)

Benzyl phenyl sulfone (2.6\%)

Benzyl phenyl sulfoxide $(0.07 \%)$

Benzoic acid $(0.28)$

42.BPS + 9-fluorenone + decalin

Benzaldehyde $(0.2$ \% $)$

Dibenzyl $(0.2 \%)$

PS (71.2\%)

BPS $(0.68)$

Benzyl phenyl sulfone (1.8\%)

Benzyl phenyl sulfoxide $(0.003 \%)$

9-Fluorenone $(7.2 \%)$

43.BPS + fluorenone + quinoline

Toluene (1.98)

Benzaldehyde $(0.8 \%)$

Benzoic acid $(0.1 \%)$

Dibenzy 1 (4.1\%)

Benzyl phenyl sulfone (11.0\%)

PS $(41.8 \%)$

9-fluorenone (2.98) 


\section{PROJEC'T MANAGEMEN'T REPOR'T}

March 1 through May 31, 1992

Project Title: Protocols for the Selective Cleavage of Carbon-Sulfur Bonds in Coal

Principal Investigator: Mark J. Bausch

Department of Chemistry \& Biochemistry Southern Illinois University at Carbondale Carbondale, Illinois 62901 (618) 453-6461

Project Monitor:

Ken K. Ho, CRSC

\section{COMMENTS}

This project is funded by the U. S. Department of Energy (PETC) and by the Illinois Department of Energy and Natural Resources as part of their costshared programs. 


\section{PROJEC'T MILES'TONES}

Protocols for the Selective Cleavage of Carbon-Sulfur Bonds in Coal

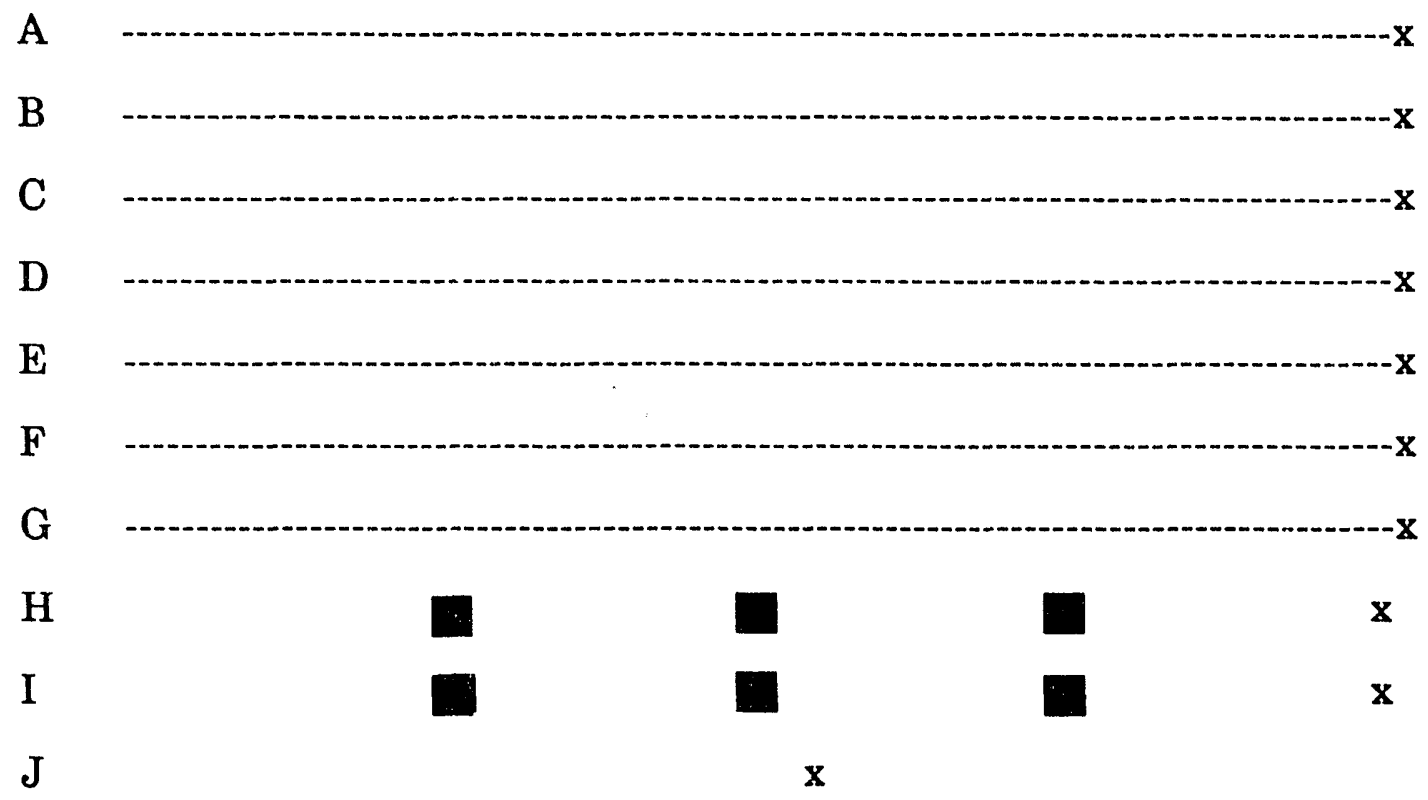

$\begin{array}{llllllllllllll}\underset{\text { Bept. 1 }}{\text { Begin }} & \text { S } & \text { O } & \text { N } & \text { D } & \text { J } & \text { F } & \text { M } & \text { A } & \text { M } & \text { J } & \text { J } & \text { A } & \text { S }\end{array}$

1991

Milestones:
A. Postdoctoral research associate employed.
B. Graduate research assistant employed.
C. Graduate research assistant employed.
D. TASK 1 undertaken; internal Bausch group TASK 1 review February $1,1992$.
E. TASK 2 undertaken; internal Bausch group TASK 2 review March 1, 1992.
F. TASK 3 undertaken; internal Bausch group TASK 3 review April 1, 1992.
G. TASK 4 undertaken; internal Bausch group TASK 4 review May 1, 1992.
H. Technical reports prepared and submitted.
I. Project management reports prepared and submitted.
J. Publication prepared and submitted: "C-S Bond Strengths in Radical Cations."

Comments: Milestone $J$ has not yet been met. 

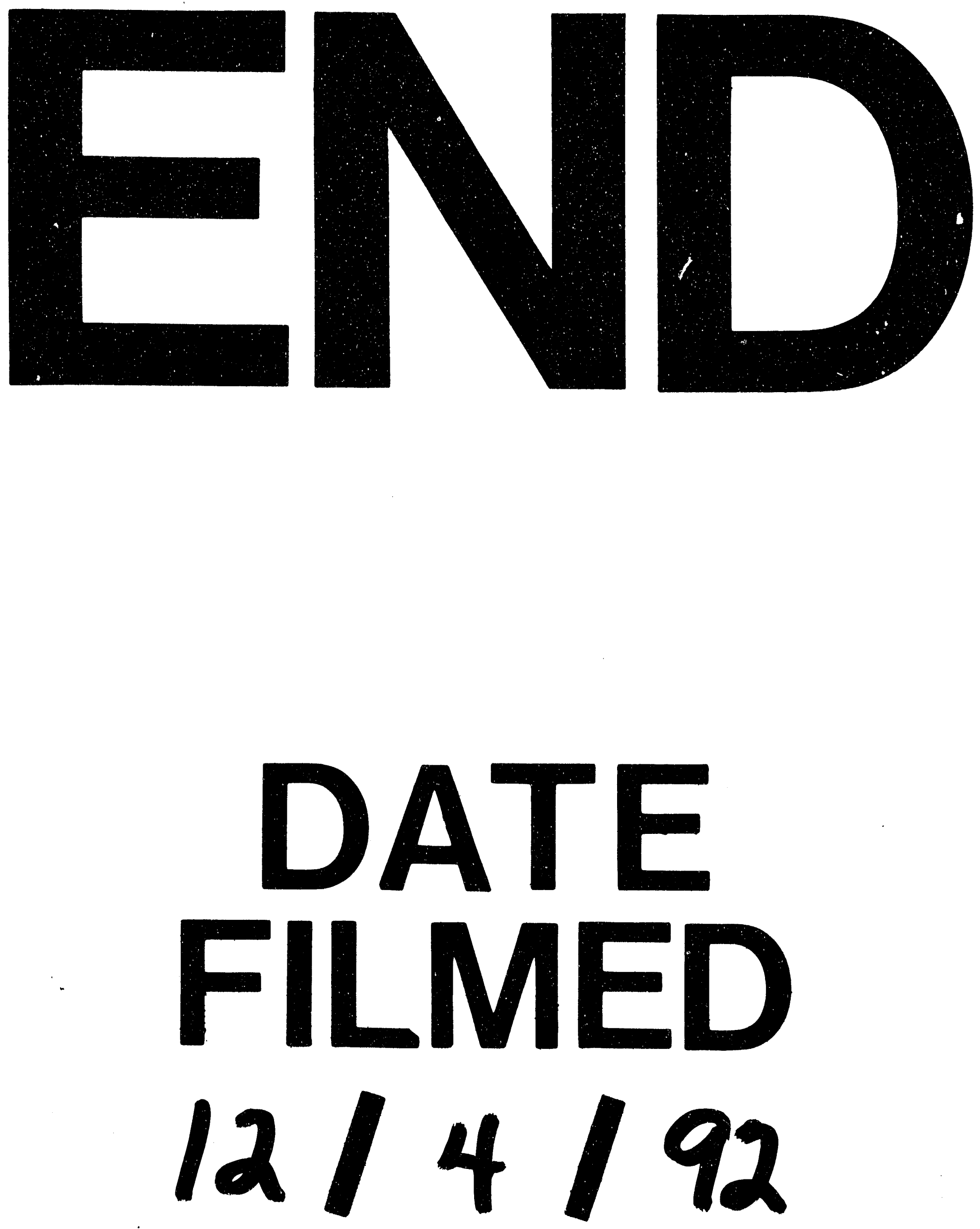
, 